\title{
A CHARACTERIZATION OF MANIFOLD DECOMPOSITIONS SATISFYING THE DISJOINT TRIPLES PROPERTY
}

\author{
DENNIS J. GARITY
}

\begin{abstract}
A metric space $X$ satisfies the Disjoint Triples Property $\left(\mathrm{DD}_{3}\right)$ if maps $f_{1}, f_{2}$ and $f_{3}$ from $B^{2}$ into $X$ are approximable by maps $\tilde{f}_{1}, \tilde{f}_{2}$ and $\tilde{f}_{3}$ with $\bigcap_{i=1}^{3} \tilde{f}_{i}\left(B^{2}\right)=\varnothing$. Those $\mathrm{CE}$ decompositions of manifolds satisfying $\mathrm{DD}_{3}$ and yielding finite-dimensional nonmanifold decomposition spaces are shown to be precisely those intrinsically 0 -dimensional decompositions which yield nonshrinkable null cellular decompositions under amalgamation. This characterization results in another proof of the fact that $E^{n} / G \times E^{1}$ is secretly 0-dimensional where $G$ is a CE usc decomposition of $E^{n}, n>4$, with $E^{n} / G$ finite dimensional.
\end{abstract}

0. Introduction. Recent work of Cannon [C], Edwards [Ed] and Quinn [Q] characterizes topological $n$-manifolds, $n \geqslant 5$, in terms of a simple general position property, the Disjoint Disks Property (DDP). Daverman introduced a property closely related to the DDP, the Disjoint Triples Property (hereafter referred to as $\mathrm{DD}_{3}$ ), and showed that certain decompositions satisfying $\mathrm{DD}_{3}$ can be amalgamated so as to yield nonshrinkable null cellular decompositions. See [D1].

We show that finite-dimensional nonshrinkable decompositions satisfying $\mathrm{DD}_{3}$ can be characterized as those intrinsically 0 -dimensional decompositions having amalgamations as above. Thus, the class of finite-dimensional decompositions satisfying $\mathrm{DD}_{3}$ but not DDP has the minimal amount of complexity required to yield nonmanifold decompositions in the sense that nonshrinkable null cellular decompositions form a prototype for this class.

It is also shown that $E^{n} / G \times E^{1}$ satisfies $\mathrm{DD}_{3}$ where $G$ is any $\mathrm{CE}$ uscd of $E^{n}$, $n \geqslant 4$. This result, combined with the above characterization, gives another proof of the fact that $E^{n} / G \times E^{1}$ is secretly 0-dimensional when $E^{n} / G$ is finite dimensional [D3, p. 133].

This paper contains results presented in my thesis [G1] completed under the supervision of J. W. Cannon.

1. Notation and preliminaries. We will be considering cell-like (CE) upper semicontinuous decompositions (uscd) $G$ of $n$-manifolds $M$. If $G$ is such a decomposition, $\pi$ or $\pi_{G}$ represents the natural quotient map from $M$ onto $M / G, H_{G}$ represents the set consisting of the nondegenerate elements of $G$, and $N_{G}$ represents

Received by the editors November 14, 1980.

1980 Mathematics Subject Classification. Primary 54F65, 54B15, 57N60, 57P95; Secondary 57N15, 54B10, 54N75.

Key words and phrases. Cell-like decomposition, cellular set, disjoint disks property, disjoint triples property, intrinsic dimension, null sequence, secret dimension, shrinkable.

(c) 1981 American Mathematical Society 0002-9939/81/0000-0583/\$02.50 
the union of these elements. Given a closed subset $A$ of $M / G, M / \pi^{-1}(A) \equiv M / G^{\prime}$ where $G^{\prime}$ is the decomposition having as elements the singletons of $M-\pi^{-1}(A)$ together with the elements of $\left\{\pi^{-1}(a) \mid a \in A\right\}$.

If $p$ is a CE map from $M$ onto $X, N_{p} \equiv N_{L}$ where $L$ is the decomposition of $M$ with elements $\left\{p^{-1}(x) \mid x \in X\right\}$. The map is said to be $1-1$ over $A \subset X$ if $p \mid p^{-1}(A)$ is 1-1. The metric on a space $X$ will be denoted by $\rho$ or $\rho_{x}$. The space $\delta$ of maps from a compact space $X$ into a complete separable metric space $Y$ will be given the topology induced by the metric $\rho_{\varsigma}(f, g) \equiv \sup _{x \in X} \rho_{Y}(f(x), g(x))$. By [K, p. 93], $\left(\mathcal{S}, p_{\delta}\right)$ is a complete separable metric space. To say that a map $f$ from $X$ into $Y$ is approximable by maps $g$ will mean, using the above notation, that for each $\varepsilon>0$ there exists a map $g$ with $\rho_{\delta}(f, g)<\varepsilon$.

Definition. Let $G$ be a CE uscd of an $n$-manifold $M$ and $f$ a map from $B^{2}$ into $M / G$. Then maps $F: B^{2} \rightarrow M$ such that $\pi \circ F$ is an approximation to $f$ are called approximate lifts of $f$.

By [L, p. 506], $f$ is always approximable by maps $\pi \circ F$ where $F$ is an approximate lift of $f$.

Defintion. Let $G$ be a CE uscd of an $n$-manifold $M$. Then $G$ is secretly $d$-dimensional if $\pi$ is approximable by CE maps $p$ from $M$ onto $M / G$ with the dimension of $p(N p) \leqslant d$. $G$ is said to be intrinsically $d$-dimensional if it is secretly $d$-dimensional, but is not secretly $(d-1)$-dimensional.

Definition. A metric space $X$ has property $\mathrm{DD}_{K}, 2<K<\infty$, if maps $f_{i}$ : $B^{2} \rightarrow X, 1 \leqslant i \leqslant K$, are approximable by maps $\tilde{f}_{i}: B^{2} \rightarrow X, 1<i<K$, with $\cap_{i=1}^{K} \tilde{f}_{i}\left(B^{2}\right)=\varnothing$.

Remark 1. Property DDP is the same as $\mathrm{DD}_{2}$. The Disjoint Triples Property is the same as $\mathrm{DD}_{3}$. A decomposition $G$ of $M$ is said to satisfy $\mathrm{DD}_{K}$ if $M / G$ satisfies $\mathrm{DD}_{K}$.

Definition. Let $G$ be a CE uscd of an $n$-manifold $M$ and let $\varepsilon>0$ be fixed. Then $Q$ is said to be an $\varepsilon$-amalgamation of $G$ if

(i) $Q$ is a $C E$ uscd of $M$,

(ii) each $g \in G$ is contained in some $a \in \mathbb{Q}$, and

(iii) for each $x \in M / Q, \pi_{G} \circ \pi_{\mathscr{Q}}^{-1}(x)$ is of diameter $<\varepsilon$.

REMARK 2. The space $M / A$ can be thought of as a CE decomposition of $M / G$.

The following technical lemma can be proved using standard properties of CE maps.

LEMMA 1. Let $G$ be a $C E$ uscd of an $n$-manifold $M, n>5$, and $A$ closed in $M / G$. If pairs of maps $\left(f_{1}, f_{2}\right): B^{2} \rightarrow M / G$ are approximable by pairs $\left(\tilde{f}_{1}, \tilde{f}_{2}\right)$ with $\tilde{f}_{1}\left(B^{2}\right) \cap$ $\tilde{f}_{2}\left(B^{2}\right) \cap A=\varnothing$, then $M / \pi^{-1}(A)$ satisfies $D D P$.

2. Spaces that satisfy $\mathbf{D D}_{\mathbf{3}}$. In this section, sufficient conditions for a decomposition to satisfy $\mathrm{DD}_{3}$ are given and results leading to the characterization of the next section are presented.

Proposition 1. Let $G$ be a $C E$ uscd of an $n$-manifold $M, n>4$, such that $H_{G}$ consists of a countable number of cellular sets. Then $M / G$ satisfies $D D_{3}$. 
Proof. Choose $\varepsilon>0$ and maps $f_{e}: B^{2} \rightarrow M / G, 1<e<3$. Choose approximate lifts $F_{1}$ and $F_{2}$ to $f_{1}$ and $f_{2}$ so that $F_{1}\left(B^{2}\right) \cap F_{2}\left(B^{2}\right)$ consists of (at most) a finite number of points, and so that $\tilde{f}_{e} \equiv \pi \circ F_{e}, e=1$ or 2 , is within $\varepsilon$ of $f_{e}$. Then $\tilde{f}_{1}\left(B^{2}\right) \cap \tilde{f}_{2}\left(B^{2}\right) \equiv A$ consists of at most countably many points in $M / G$.

Since $G$ is cellular, an $\varepsilon$ approximation $\tilde{f}_{3}$ to $f_{3}$ can be found so that $\tilde{f}_{3}\left(B^{2}\right) \cap A=$ $\varnothing$. The required approximations are $\tilde{f}_{1}, \tilde{f}_{2}$ and $\tilde{f}_{3}$.

The examples of [D1, W and DW] are of nonshrinkable decompositions satisfying the hypotheses of Proposition 1, thus showing that there exist nonmanifold spaces satisfying $\mathrm{DD}_{3}$.

Proposition 2. A complete separable metric, $A N R X$, satisfies $D D_{3}$ if and only if each map $f$ from $B^{2}$ into $X$ is approximable by maps $\tilde{f}$ such that $\left\{\tilde{f}^{-1}(x)\right\}$ has cardinality less than 3 for each $x$ in $X$.

Proof. The proof of the forward implication is modeled after the argument presented in [C, Theorem 2.1]. Let $\left\{\left(\boldsymbol{P}_{i}(1), \boldsymbol{P}_{i}(2), \boldsymbol{P}_{i}(3)\right)\right\}, 1<i<\infty$, be a countable collection of triples of subdiscs of $B^{2}$ chosen so that:

(1) for each $i, P_{i}(j) \cap P_{i}(k)=\varnothing$ for $j \neq k$; and

(2) given distinct points $x_{1}, x_{2}$ and $x_{3}$ in $B^{2}$, there exists an $i$ such that $x_{e} \in P_{i}(e)$, $1<e \leqslant 3$.

Choose $\varepsilon>0$ and $f: B^{2} \rightarrow X$. Since $X$ satisfies $\mathrm{DD}_{3}, \theta_{i} \equiv\left\{h: B^{2} \rightarrow X \mid\right.$ $\left.\cap_{e=1}^{3} h\left(P_{i}(e)\right)=\varnothing\right\}$ is dense and open in the space of all maps from $B^{2}$ into $X$. It follows from the Baire Category Theorem [CV, p. 89] that there exists a map $\tilde{f}$ in $\cap_{i=1}^{\infty} \theta_{i}$ with $\rho(f, \tilde{f})<\varepsilon$. The map $\tilde{f}$ is the required approximation to $f$.

For the reverse implication, choose $\varepsilon>0$ and maps $f_{e}: B^{2} \rightarrow X, 1 \leqslant i<3$. Choose arcs $h$ joining $f_{1}\left(\partial B^{2}\right)$ to $f_{2}\left(\partial B^{2}\right)$ and $l$ joining $f_{2}\left(\partial B^{2}\right)$ to $f_{3}\left(\partial B^{2}\right)$. Then $\left(\cup_{e=1}^{3} f_{e}\left(B^{2}\right)\right) \cup h \cup l$ can be viewed as the image of a map $F$ from $B^{2}$ into $X$, with subdiscs $D_{e}, 1 \leqslant e \leqslant 3$, of $B^{2}$ so that $F \mid D_{e}$ is an $\varepsilon / 3$ approximation to $f_{e}$.

By assumption, $F$ can be $\varepsilon / 3$ approximated by a map $\tilde{f}$ so that each point of $X$ has less than 3 preimages under $\tilde{f}$. The required approximations to $f_{e}, 1<e<3$, are the $\tilde{f} \mid D_{e}$.

Corollary 1. Let $G$ be a $C E$ uscd of an $n$-manifold $M, n \geqslant 5$, so that $M / G$ is an $A N R$ and satisfies $D D_{3}$. Then $G$ is secretly 0-dimensional.

Proof. Let $\left\{\left(F_{i}, H_{i}\right)\right\}, 1 \leqslant i<\infty$, be a countable dense subset of the space of pairs of maps from $B^{2}$ into $M / G$. The argument used in the proof of Proposition 2 allows one to require that, for each $i, F_{i}^{-1}(x) \cup H_{i}^{-1}(x)$ has cardinality less than 3 for each $x \in M / G$.

Thus, each $H_{i}\left(B^{2}\right)$ is the, at most, 2-to-1 image of a 2-dimensional space, and so has dimension $\leqslant 3$ [En, p. 134]. This allows one to further require that, for each $i, F_{i}^{-1}\left(H_{i}\left(B^{2}\right)\right)$ is 0-dimensional [C, Theorem 2.2]. Thus, $F_{i}\left(B^{2}\right) \cap H_{i}\left(B^{2}\right)$ is the 1-1 image of a 0-dimensional set and so has dimension 0 [En, p. 134]. Let $A=$ $\cup_{i=1}^{\infty}\left[F_{i}\left(B^{2}\right) \cap H_{i}\left(B^{2}\right)\right]$.

The set $A$ is a 0 -dimensional $F \sigma$, and so by [En, p. 45], there exist a 0 -dimensional $G_{\delta}, L$, with $A \subset L$. Let $C$ be any closed set in $M / G-L$. Lemma 1 implies 
that $M / \pi^{-1}(C)$ satisfies the DDP, so by [Ed], $p: M \rightarrow M / \pi^{-1}(C)$ is approximable by homeomorphisms. By [Ev, p. 15], it follows that $\pi: M \rightarrow M / G$ is approximable by a CE map $q$ that is 1-1 over $M / G-L$. It follows that $q\left(N_{q}\right)$ is contained in the 0 -dimensional set $L$, and so $G$ is secretly 0 -dimensional.

Proposition 3. Let $G$ be a $C E$ uscd of an $n$-manifold $M, n>4$. Then $(M / G \times$ $\left.E^{1}\right)$ satisfies $D_{3}$.

Proof. Choose $\varepsilon>0$ and maps $f_{e}: B^{2} \rightarrow\left(M / G \times E^{1}\right), 1<e<3$. Let $p$ be the projection of $M / G \times E^{1}$ onto $M / G$ and $q$ the projection onto $E^{1}$. Choose a triangulation $T$ of $B^{2}$ so that for each $\sigma \in T$, and for $1 \leqslant e<3$, there exist open sets $V_{e}(\sigma)$ and $W_{e}(\sigma)$ in $M / G$, and open intervals $K_{e}(\sigma)$ and $J_{e}(\sigma)$ in $E^{1}$ satisfying:

(1) $f_{e}(\sigma) \subset V_{e}(\sigma) \times K_{e}(\sigma) \subset W_{e}(\sigma) \times J_{e}(\sigma)$;

(2) $W_{e}(\sigma) \times J_{e}(\sigma)$ has diameter $<\varepsilon / 3$; and

(3) $V_{e}(\sigma)$ contracts in $W_{e}(\sigma)$.

By [D2, Lemma 2.6], there exists $\varepsilon / 3$ approximations $h_{e}$ to $f_{e}, 1<e<3$, so that

(4) $\left(p \circ h_{e} \mid T^{1}\right) \cap\left(p \circ h_{d} \mid T^{1}\right)=\varnothing$ for $e \neq d$, and

(5) for each $\sigma$ in $T, h_{e}(\sigma) \subset V_{e}(\sigma) \times K_{e}(\sigma)$.

Let $P_{1}, P_{2}$ and $P_{3}$ be pairwise disjoint dense subsets of $E^{1}$. For each two cell $\sigma \in T$, let $C(\sigma)$ be a small interior collar on $\partial \sigma$. Let $N(\sigma)$ represent the interior boundary of $C(\sigma), C=\cup_{\sigma \in T} C(\sigma)$, and $U=B^{2}-C$.

Focus attention on a specific two cell $\sigma$. Define an approximation $\tilde{f}_{e}$ to $h_{e}$, $1 \leqslant e \leqslant 3$, as follows:

(6) $\tilde{f}_{e} \mid C(\sigma)$ is defined so that $p \circ \tilde{f}_{e}(C(\sigma))=p \circ h_{e}(\partial \sigma)$;

(7) $q \circ \tilde{f}_{e}(N(\sigma)) \equiv a_{e}(\sigma)$, a point in $P_{e} \cap K_{e}(\sigma)$;

(8) $\tilde{f}_{e} \mid C(\sigma)$ is extended to all of $\sigma$ using the contraction of $V_{e}(\sigma)$ in $W_{e}(\sigma)$ so that $q \circ \tilde{f}_{e}(\sigma-C(\sigma))=a_{e}(\sigma)$.

Condition (6) guarantees that $\left[p\left(\tilde{f}_{e}(C)\right)\right] \cap\left[p\left(\tilde{f}_{d}(C)\right)\right]=\varnothing$ for $e \neq d$. Condition (8) guarantees that $\left[q\left(\tilde{f}_{e}(U)\right)\right] \cap\left[q\left(\tilde{f}_{d}(U)\right)\right]=\varnothing$ for $e \neq d$. It follows that $\cap_{e=1}^{3} \tilde{f}_{e}\left(B^{2}\right)=\varnothing$ and that $M / G \times E^{1}$ satisfies $\mathrm{DD}_{3}$.

Theorem 1 [D3, p. 133]. Let $G$ be as in Propositions 3 with $M / G$ finite dimensional. Then $M / G \times E^{1}$ is secretly 0-dimensional.

Proof. This follows directly from Corollary 1 and Proposition 3.

3. The characterization. The following amalgamation lemma can be found in slightly different form in [D1, p. 173 and Ed]. It has shown its importance in decomposition theory, both in dimension 4 and in dimensions $>5$. See [DP, DR and Ed].

Amalgamation Lemma. Let $G$ be a $C E$ uscd of an $n$-manifold $M, n \geq 3$, so that $\pi(N \pi)$ is 0-dimensional. Let $F=\cup_{i=1}^{\infty} F_{i}$ be an Fo set in $M / G-\pi\left(N_{G}\right)$ of dimension $\leqslant n-2$. Then, for each $\varepsilon>0$, there exists an $\varepsilon$-amalgamation $A(\varepsilon)$ of $G$ such that

(1) $H_{A(e)}$ consists of a null sequence and

(2) $N_{A(\varepsilon)} \cap \pi^{-1}(F)=\varnothing$. 
The next lemma follows from Bing's Shrinking Criterion [B]. For a proof, see [D1, p. 174 or Ed].

LEMMA 3. Let $G$ be a $C E$ uscd of an $n$-manifold $M, n>5$, such that for every $\varepsilon>0$ there exists an $\varepsilon$-amalgamation $A(\varepsilon)$ of $G$ with $M / A(\varepsilon) \cong M$. Then $M / G \cong$ M.

TheOrem 2 (ChARACTERIZATION TheOREM). Let $G$ be a $C E$ uscd of an $n$-manifold $M, n \geqslant 5$, with $M / G$ an $A N R$. Then $M / G$ satisfies $D D_{3}$ but not $D D P$ if and only if

(1) $G$ is intrinsically 0-dimensional, i.e. there exists a $C E$ uscd $G^{\prime}$ of $M$ with $M / G \cong M / G^{\prime} \approx M$, and $\pi_{G^{\prime}}\left(N_{G^{\prime}}\right)$ of dimension 0 , and

(2) for each $\varepsilon>0$, there exists an $\varepsilon$-amalgamation $A(\varepsilon)$ of $G^{\prime}$ with $H_{A(\varepsilon)}$ consisting of a null sequence of cellular sets, and $M / A(\varepsilon) \approx M$.

Proof. Part of the proof of the forward implication can be found in [D1, p. 175]. For completeness, the entire proof is presented. Assume that $M / \tilde{G}$ satisfies $\mathrm{DD}_{3}$ but not DDP. Then by Corollary 1 and [Ed], there exists a decomposition $\tilde{G}$ of $M$ with $\pi_{\tilde{G}}\left(N_{\tilde{G}}\right)$ of dimension 0 , and $M / \tilde{G} \cong M / G \approx M$. Let $\left\{F_{i}\right\}, 1 \leqslant i<\infty$, be a countable dense subset of the space of maps from $B^{2}$ into $M / G$. By an argument similar to that used in the proof of Proposition 2, we can require that, for each $x \in M / G, \cup_{i=1}^{\infty} F_{i}^{-1}(x)$ has cardinality less than 3 . Let $p$ be the projection map from $M$ onto $M / \tilde{G}$.

Each $M / p^{-1}\left(F_{i}\left(B^{2}\right)\right)$ satisfies the DDP by Lemma 1. It follows from [Ed and Ev, p. 15] that $p$ is approximable by a CE map $q$ from $M$ onto $M / \tilde{G}$, such that $q$ is 1-1 over $\cup_{i=1}^{\infty} F_{i}\left(B^{2}\right)$ and over $M / \tilde{G}-S$ where $S$ is a 0 -dimensional set containing $p\left(N_{\tilde{G}}\right)$. Let $G^{\prime}$ be the decomposition such that $N_{G^{\prime}}=N_{q}$. Then $G^{\prime}$ satisfies (1).

By [En, p. 134], each $F_{i}\left(B^{2}\right)$ has dimension less than or equal to 3. (In fact, the $F_{i}$ can be chosen so that each $F_{i}\left(B^{2}\right)$ has dimension $=2$. See [D2, Theorem 3.3].) Let $F=\cup_{i=1}^{\infty} F_{i}\left(B^{2}\right)$. Then $F$ is an at most 3-dimensional $F \sigma$ set. So the amalgamation lemma can be applied to obtain for each $\varepsilon$, an $\varepsilon$-amalgamation $A(\varepsilon)$ of $G^{\prime}$, so that $H_{A(\varepsilon)}$ consists of a null sequence, and so that $P\left(N_{A(\varepsilon)}\right) \cap F=\varnothing$. Lemma 3 allows one to assume that $M / A(\varepsilon) \nRightarrow M$. It remains to check that $A(\varepsilon)$ is cellular.

Each point $x$ in $M / A(\varepsilon)$ satisfies McMillan's Cellularity Criterion [M, p. 328] since $\pi_{A(\varepsilon)}\left(N_{A(\varepsilon)}\right) \cap \pi_{A(\varepsilon)}(F)=\varnothing$. This implies that each element of $A(\varepsilon)$ satisfies McMillan's Cellularity Criterion in $M$, and so by [M, p. 328], $A(\varepsilon)$ is cellular.

For the proof of the reverse implication, assume that (1) and (2) hold. By [Ed], $G$ fails to satisfy the DDP. Choose $\varepsilon>0$ and maps $f_{e}, 1 \leqslant e \leqslant 3$, from $B^{2}$ into $M / G$. Let $A$ be an $\varepsilon / 4$ amalgamation of $G^{\prime}$ as in (2). Let $p=\pi_{A} \circ \pi_{G}^{-1}$. For each $x$ in $M / G$, let $U(x)$ be the $\varepsilon / 2$ neighborhood of $x$. The fact that $G$ and $A$ are uscd, along with the choice of $A$, yields the result that each $p(U(x))$ contains a nonempty open set $V(x)$.

Cover $\cup_{e=1}^{3} f_{e}\left(B^{2}\right)$ by a finite number of these sets, $V_{1}, \ldots, V_{s}$. Let $\delta=$ the Lebesgue number of the cover $\left\{\left(V_{i}\right)\right\}, 1 \leqslant i \leqslant s$, of $\cup_{e=1}^{3} f_{e}\left(B^{2}\right)$. Proposition 1 implies that there exist $\delta / 3$ approximations $F_{e}$ to $p \circ f_{e}, 1 \leqslant e \leqslant 3$, so that $\cap_{e=1}^{3} F_{e}\left(B^{2}\right)=\varnothing$. Choose approximate lifts $\tilde{f}_{e}: B^{2} \rightarrow M / G$ to the $F_{e}$ so that 
$p\left(p \circ \tilde{f}_{e}, F_{e}\right)<\delta / 3$, and so that $\cap_{e=1}^{3} p \circ \tilde{f}_{e}\left(B^{2}\right)=\varnothing$. It follows that the $\tilde{f}_{e}$ are $\varepsilon$ approximations to the $f_{e}, 1 \leqslant e \leqslant 3$, with $\cap_{e=1}^{3} \tilde{f}_{e}\left(B^{2}\right)=\varnothing$. This completes the proof.

REMARK 3. The characterization given in Theorem 2 is nontrivial in the following sense. Not all cellular decompositions of $M$ are intrinsically 0-dimensional [DG] and there exist 0 -dimensional cellular decompositions failing to satisfy $\mathrm{DD}_{3}$ [G2].

\section{REFERENCES}

[B] R. H. Bing, Upper semicontinuous decompositions of $E^{3}$, Ann. of Math. (2) 65 (1957), 363-374.

[C] J. W. Cannon, Shrinking cell-like decompositions of manifolds. Codimension three, Ann. of Math. (2) 110 (1979), 83-112.

[CV] C. D. Christenson and W. L. Voxman, Aspects of topology, Marcel Dekker, New York, 1977.

[D1] R. J. Daverman, A nonshrinkable decomposition of $S^{n}$ determined by a null sequence of cellular sets, Proc. Amer. Math. Soc. 75 (1979), 171-176.

[D2] __, Detecting the disjoint disks property, Pacific J. Math. (to appear).

[D3] __ Products of cell-like decompositions, Topology Appl. 11 (1980), 121-139.

[DG] R. J. Daverman and D. J. Garity, Intrinsically $(n-2)$-dimensional cellular decompasitions of $E^{n}$ (preprint).

[DP] R. J. Daverman and D. K. Preston, Cell-like 1-demensional decompositions of $S^{3}$ are 4-manifold factors, Houston J. Math. 6 (1980), 491-502.

[DR] R. J. Daverman and W. H. Row, Cell-like 0-dimensional decompasitions of $S^{3}$ are 4 -manifold factors, Trans. Amer. Math. Soc. 254 (1979), 217-236.

[DW] R. J. Daverman and J. J. Walsh, $A$ nonshrinkable decomposition of $S^{n}$ involving a mull sequence of cellular arcs, Trans. Amer. Math. Soc. (to appear).

[En] R. Engelking, Dimension theory, North-Holland, Amsterdam, 1978.

[Ed] R. D. Edwards, Approximating certain cell-like maps by homeomorphisms, (preprint); Notices Amer. Math. Soc. 24 (1977), A-649; Abstract \#751-G5.

[Ev] D. L. Everett, Embedding and product theorems for decompositions spaces, Doctoral Thesis, University of Wisconsin, Madison, 1976.

[G1] D. J. Garity, General position properties of homology manifolds, Doctoral Thesis, University of Wisconsin, Madison, 1980.

[G2] __, General position properties related to the Disjoint Discs Property (preprint).

[K] K. Kuratowski, Topology. Vol. II, Academic Press, New York, 1968.

[L] R. C. Lacher, Cell-like mappings and their generalizations, Bull. Amer. Math. Soc. 83 (1977), 495-552.

[M] D. R. McMillan Jr., A criterion for cellularity in a manifold, Ann. of Math. (2) 79 (1964), 327-337.

[Q] F. Quinn, Ends of maps. I, Ann. of Math. (2) 11 (1979), 275-331.

[W] D. G. Wright, $A$ decomposition of $E^{n}(n>3)$ into points and a mull sequence of cellular sets, General Topology Appl. 10 (1979), 297-304.

Department of Mathematics, University of Tennessee, Knoxville, Tennessee 37916

Current address: Department of Mathematics, Oregon State University, Corvallis, Oregon 97331 